\title{
Genetics, Epigenetics and Leukemia
}

\author{
Kevin Shannon, M.D. ${ }^{1}$ and Scott A. Armstrong, M.D., Ph.D. ${ }^{2}$ \\ ${ }^{1}$ Department of Pediatrics and Comprehensive Cancer Center, University of California, San \\ Francisco, California \\ ${ }^{2}$ Department of Pediatric Oncology, Dana-Farber Cancer Institute, Department of Medicine, \\ Children's Hospital Boston, Harvard Medical School, Boston, Massachusetts
}

In this issue of the Journal, Ley and colleagues ${ }^{1}$ report that somatic mutations in DNMT3A are common in adults with acute myelogenous leukemia (AML), are associated with discrete molecular and cytogenetic features, and have prognostic significance. These findings have implications for clinical investigators, for researchers working to characterize the molecular mechanisms that contribute to leukemic growth, and, more broadly, for illuminating mechanisms of epigenetic reprogramming in cancer cells.

Ley et al. sequenced the genome of leukemia cells from a person with AML, and observed a DNMT3A mutation. They went on to analyze a large and well-annotated panel of adult AMLs, of which $22 \%$ turned out to harbor a somatic DNMT3A mutation. Most AML patients with DNMT3A mutations were classified as having an intermediate risk of treatment failure based on cytogenetic features at diagnosis; often, their leukemias also harbored mutations in other genes that are altered commonly in AML (IDH1, IDH2, FLT3, and/or $N P M$ ). The authors did not detect DNMT3A mutations in leukemias with a PML-RARA translocation or other translocations associated with a relatively favorable clinical outcome. Most importantly for clinicians who care for patients with AML, the authors showed that mutations in DNMT3A are a strong and independent predictor of relapse.

These new data and a previous investigation of $\mathrm{AML}^{2}$ underscore the power of whole genome sequencing studies to uncover new molecular lesions in cancer, and support the sequencing of AML genomes of patients with "high risk" disease, for which there is a paucity of molecular data.

Because Ley and colleagues analyzed archival specimens from patients who received similar anti-cancer therapies, they were able to perform statistical analyses to determine if mutation status affected outcome. However, more research is required before these results can be directly translated into clinical practice. The prevalence and prognostic significance of DNMT3A mutations in AML must now be determined in additional patients; the prevalence reported by Ley et al. is higher than was observed in a smaller series, albeit using a different

Corresponding Author: Kevin Shannon, M.D., Department of Pediatrics, University of California, Helen Diller Family Cancer Research Building, 1450 3rd Street; Room 240, San Francisco, CA 94158-9001, TEL: 415 476-7932, FAX: 415 502-5127, shannonk@peds.ucsf.edu.

Disclosure: Disclosure forms provided by the authors are available with the full text of this article at NEJM.org. 
approach. ${ }^{3}$ Analyzing pediatric AML samples will also be important; it's conceivable that the presence of a DNMT3A mutation in this context could inform the decision of when to perform allogeneic hematopoietic stem cell transplantation (HSCT) during first remission. An analysis carried out by Ley et al. ${ }^{3}$ suggested that patients with DNMT3A mutations who received HSCT had better outcomes. Finally, as inhibitors of mutant proteins such as FLT3 and other new therapies are tested in AML, it will be essential to continually reassess the impact of DNMT3A mutations on response and resistance.

The DNMT3A protein is a member of a family of methyltransferase enzymes that catalyze the addition of methyl groups to DNA where the sequence contains $\mathrm{CpG}$ dinucleotides. DNA methylation at $\mathrm{CpG}$ islands located near genes regulates gene expression. DNMT3A and DNMT3B initiate most DNA methylation, while DNMT1 maintains a stable methylation pattern. The DNMT3A mutations found in AML fall into two classes: those likely to abrogate protein function and those that effect an amino-acid substitution of arginine at position 882 of the protein (R882). The clinical and biologic features of patients with both types of mutation are similar, with the exception that patients with R882 mutations had higher blood leukocyte counts at diagnosis. Amino-acid substitutions at R882 impair, but do not eliminate, DNMT3A methyltransferase activity. ${ }^{3}$ In the vast majority of patients, a single DNMT3A allele is mutated, consistent with the observation of residual DNMT3A expression in every AML examined by Ley and colleagues.

What are the likely functional consequences of these somatic DNMT3A mutations? The most plausible model is that DNMT3A is a tumor suppressor gene, and that its mutation contributes to leukemogenesis and/or drug resistance by reducing global DNMT3A methyltransferase activity. This, in turn, could result in de-repression of one or more genes critical to the normal balance of self-renewal, proliferation, and differentiation of hematopoietic stem cells (HSCs). Consistent with this idea are recent studies showing that DNA methylation profiles change as blood cells develop from HSCs, and that ablation of Dnmt1 in mice perturbs HSC self-renewal and hematopoietic cell-fate decisions. ${ }^{4-6}$ Ley et al. did not observe DNMT3A mutations in AMLs with translocations involving genes (such as MLL and RARA) that regulate HSC fate by modulating gene expression. Perhaps each of these alterations impairs differentiation and leads to unregulated self-renewal/proliferation via mechanisms that impinge upon overlapping pathways. The absence of global changes in DNA methylation in DNMT3A-mutated AMLs indicates that establishing how these mutations contribute to leukemogenesis will require much work.

Advances in understanding the molecular pathogenesis of AML have yet to be translated into more effective and less toxic treatments; the therapeutic "backbone" of intensive cytosine arabinoside and anthracycline chemotherapy has not changed over thirty years. It is unclear how the finding of DNMT3A mutations in AML might lead to novel therapeutic approaches. Like other loss-of-function mutations in cancer, DNMT3A mutations do not result in the production of a "druggable" protein. These mutations likely contribute to leukemic growth by modulating a highly interdependent network of epigenetic processes such as DNA methylation, histone methylation, and histone acetylation. As we learn more about how such mutations deregulate cell fates, it may prove feasible to develop drugs that reverse the aberrant epigenetic programs in AML and other cancers. Clinical trials of DNA 
methyltransferase inhibitors and histone deacetylase inhibitors are underway in AML, and it now becomes critical to assess whether the presence of a DNMT3A mutation influences clinical responses to these agents.

The discovery of DNMT3A mutations in AML and recent reports of mutations in genes encoding histone-modifying enzymes in cancers ${ }^{7-10}$ suggest that the global patterns of aberrant epigenetic modifications seen in some cancers may result from acquired mutations in genes that control this process. That these mutations are found in primary cancer cells implies that aberrant methylation contributes directly to tumor growth rather than reflecting the maturation state of the tumor-initiating cell. Extended screening of malignancies for mutations in DNMT3A and other genes that encode enzymes involved in reversibly modifying chromatin will likely provide new insights into to mechanisms of epigenetic reprogramming in cancer, and may inform innovative therapeutic approaches to reverse the underlying aberrant gene expression programs.

\section{References}

1. Ley T. NEJM. 2010

2. Mardis ER, et al. Recurring mutations found by sequencing an acute myeloid leukemia genome. $\mathrm{N}$ Engl J Med. 2009; 361:1058-1066. NEJMoa0903840 [pii]. 10.1056/NEJMoa0903840 [PubMed: 19657110]

3. Yamashita Y, et al. Array-based genomic resequencing of human leukemia. Oncogene. 29:37233731. onc2010117 [pii]. 10.1038/onc.2010.117 [PubMed: 20400977]

4. Ji H, et al. Comprehensive methylome map of lineage commitment from haematopoietic progenitors. Nature. 467:338-342. nature09367 [pii]. 10.1038/nature09367 [PubMed: 20720541]

5. Trowbridge JJ, Snow JW, Kim J, Orkin SH. DNA methyltransferase 1 is essential for and uniquely regulates hematopoietic stem and progenitor cells. Cell Stem Cell. 2009; 5:442-449. S1934-5909(09)00400-7 [pii]. 10.1016/j.stem.2009.08.016 [PubMed: 19796624]

6. Broske AM, et al. DNA methylation protects hematopoietic stem cell multipotency from myeloerythroid restriction. Nat Genet. 2009; 41:1207-1215. ng.463 [pii]. 10.1038/ng.463 [PubMed: 19801979]

7. Nikoloski G, et al. Somatic mutations of the histone methyltransferase gene EZH2 in myelodysplastic syndromes. Nat Genet. 42:665-667. ng.620 [pii]. 10.1038/ng.620 [PubMed: 20601954]

8. van Haaften G, et al. Somatic mutations of the histone H3K27 demethylase gene UTX in human cancer. Nat Genet. 2009; 41:521-523. ng.349 [pii]. 10.1038/ng.349 [PubMed: 19330029]

9. Morin RD, et al. Somatic mutations altering EZH2 (Tyr641) in follicular and diffuse large B-cell lymphomas of germinal-center origin. Nat Genet. 42:181-185. ng.518 [pii]. 10.1038/ng.518 [PubMed: 20081860]

10. Ernst T, et al. Inactivating mutations of the histone methyltransferase gene EZH2 in myeloid disorders. Nat Genet. 42:722-726. ng.621 [pii]. 10.1038/ng.621 [PubMed: 20601953] 